\title{
MINOR MERGER ORIGIN FOR THE CIRCUMNUCLEAR STARBURST IN NGC 7742
}

\author{
L. M. Mazzuca, ${ }^{1}$ M. Sarzi, ${ }^{2}$ J. H. Knapen, ${ }^{2}$ S. Veilleux, ${ }^{3}$ and R. Swaters ${ }^{3}$ \\ Received 2006 July 10; accepted 2006 August 16; published 2006 September 15
}

\begin{abstract}
We present an emission-line diagnostic analysis of integral-field spectroscopic observations that cover the central kiloparsec of NGC 7742. This Sa galaxy hosts a spectacular nuclear starburst ring and nuclear regions characterized by low-ionization emission. The gas in the ring rotates in the opposite sense to the stars in the galaxy, suggesting a recent merging or acquisition event. The combination of integral-field measurements for the $\mathrm{H} \alpha+[\mathrm{N}$ II] emission lines from DensePak and the $\mathrm{H} \beta$ and [O III] emission from SAURON allow the construction of diagnostic diagrams that highlight the transition from star formation in the nuclear ring to excitation by highvelocity shocks or by an active galactic nucleus toward the center. DensePak measurements for the [S II] line ratio reveal very low gas densities in the nuclear ring, $N_{e}<100 \mathrm{~cm}^{-3}$, characteristic of massive $\mathrm{H}$ II regions. Comparison with MAPPINGS III models for starbursts with low gas densities show that the ring is of roughly solar metallicity. This suggests that the gas in the nuclear ring originated in a stellar system capable of substantially enriching the gas metallicity through sustained star formation. We propose that NGC 7742 cannibalized a smaller galaxy rich in metal-poor gas, and that star formation episodes in the ring have since increased the metallicity to its present value. The techniques explored here can be widely used to study similar systems, including composite (AGN+starburst) galaxies.
\end{abstract}

Subject headings: galaxies: nuclei — galaxies: spiral — galaxies: starburst — galaxies: structure

\section{INTRODUCTION}

Line emission from galaxies is produced by gas heated and ionized by hot OB stars, active galactic nuclei (AGNs), or shocks. To help interpret the spectral properties of massive star-forming regions and to recognize the role of the various excitation mechanisms, one can utilize diagnostic diagrams. Veilleux \& Osterbrock (1987, hereafter VO87) introduced a diagnostic analysis that is independent of reddening and is based on the ratios of strong emission lines rather close in wavelength. Kewley et al. (2001, hereafter K01) expand on the VO87 analysis by incorporating in their photoionization code the spectral energy distributions of young stellar clusters predicted by stellar population synthesis models (Starburst 99, Leitherer et al. 1999; PEGASE, Fioc \& Rocca-Volmerange 1997). The resulting model grids can discriminate reliably between galaxies hosting an AGN or a nuclear starburst and, in the case of $\mathrm{H}$ II regions, can constrain the metallicity of the starburst and the strength of the ionizing radiation relative to the density of hydrogen atoms (i.e., the ionization parameter, $q$ ).

NGC 7742 is a face-on SA(r)b LINER galaxy, whose morphology is dominated by a prominent nuclear ring with a radius of $10^{\prime \prime}$ or (assuming $D=22 \mathrm{Mpc}$ ) $1.0 \mathrm{kpc}$ (Mazzuca et al. 2006). The ring is lined with $H$ II regions that emit strongly in $\mathrm{H} \alpha$ (Pogge \& Eskridge 1993), but the $\mathrm{H} \alpha$ emission equivalent widths of the $\mathrm{H}$ II regions show no evidence for age gradients, indicating random star formation (Mazzuca et al. 2006). The Palomar survey (Ho et al. 1997) classified the nucleus as a T2/L2 intermediate type, where the emission is representative of a mixture of low-ionization lines (i.e., LINER) and lines associated with star formation (H II region). The galaxy is not obviously interacting at the moment but may well form a pair with NGC 7743, which is 50' (320 kpc) away but

\footnotetext{
${ }^{1}$ NASA Goddard Space Flight Center, Greenbelt, MD 20771.

${ }^{2}$ Centre for Astrophysics Research, University of Hertfordshire, Hatfield, Herts AL10 9AB, UK.

${ }^{3}$ Department of Astronomy, University of Maryland, College Park, MD 20742.
}

within $50 \mathrm{~km} \mathrm{~s}^{-1}$ in radial velocity. De Zeeuw et al. (2002; see also Sil'chenko \& Moiseev 2006) show that the gas in NGC 7742 is counterrotating with respect to the stars within the entire field of the SAURON observations. This suggests a recent merging or acquisition event.

The presence of a nuclear starburst ring and an active nucleus of composite nature in NGC 7742 makes this object particularly attractive for integral-field spectroscopy, which can capture the superposition of $\mathrm{H}$ II region and AGN emission-line features. Using the model-based diagnostic diagrams of VO87 and K01, we aim to delineate the gas conditions in the nucleus and the nuclear ring.

\section{OBSERVATIONS AND DATA REDUCTION}

In this study we combined data from two integral-field units (IFUs), namely, DensePak (Barden \& Wade 1988) on the WIYN telescope and SAURON (Bacon et al. 2001) on the William Herschel Telescope (WHT). The combined data set provides us with the key emission lines, $\mathrm{H} \beta \lambda 4861$, [O III] $\lambda \lambda 4959,5007,[\mathrm{O}$ I] $\lambda 6300,[\mathrm{~N} \mathrm{II}] \lambda \lambda 6548,6583, \mathrm{H} \alpha \lambda 6563$, and $\left[\mathrm{S}_{\mathrm{II}}\right] \lambda \lambda 6716,6731$, which are necessary to produce the diagnostic diagrams of VO87 and to constrain the gas density. The SAURON data are from Falcón-Barroso et al. (2006), which cover $\mathrm{H} \beta \lambda 4861$ and [O III] $\lambda \lambda 4959,5007$; the DensePak data (L. M. Mazzuca et al. 2006, in preparation) provide the other lines. Although both instruments are IFUs with similar fields of view $\left(33^{\prime \prime} \times 41^{\prime \prime}\right.$ and $30^{\prime \prime} \times 45^{\prime \prime}$ for SAURON and DensePak, respectively) the designs are very different. SAURON is a fully sampled lenslet array, whereas DensePak consists of 89 functional fibers (2".812 diameter) bonded into a $7 \times 13$ staggered rectangle with center-to-center fiber spacings of 3".75 and 3".25, horizontally and vertically. For NGC 7742, SAURON was configured in low-resolution mode, for a spectral resolution of $4.2 \AA$ (FWHM, $\sigma_{\text {inst }}=$ $108 \mathrm{~km} \mathrm{~s}^{-1}$ ). DensePak used the number 860 grating centered around $\mathrm{H} \alpha$, which yields a spectral resolution of $0.97 \AA$ (FWHM, $\sigma_{\text {inst }}=19 \mathrm{~km} \mathrm{~s}^{-1}$ ).

To combine the two data sets, the DensePak spectra were 

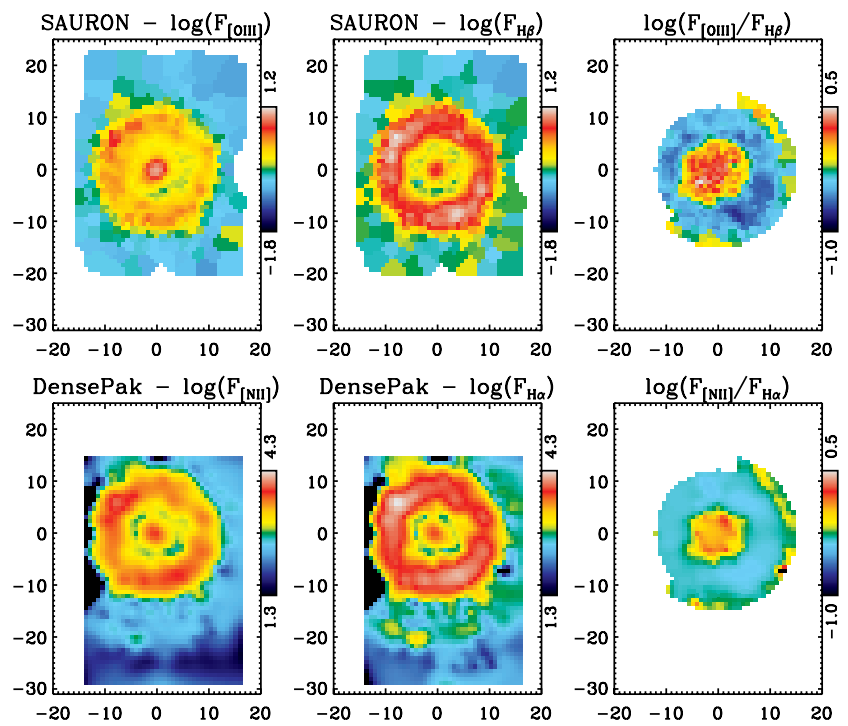

FIG. 1.-SAURON and DensePak maps for [O $\mathrm{III}]$ and $\mathrm{H} \beta$ (top left and middle panels), and for [ $\mathrm{N} \mathrm{II}]$ and $\mathrm{H} \alpha$ emission (bottom left and middle panels), together with maps over a restricted common area (to avoid regions where the DensePak coverage is poor) for the [O III] $\lambda 5007 / \mathrm{H} \beta$ and [N II] $\lambda 6583 /$ $\mathrm{H} \alpha$ line ratios (right panels). Axes are labeled with offsets in right ascension and declination (in arcseconds), with north up and east to the left.

analyzed using the procedure of Sarzi et al. (2006), in order to separate the stellar absorption and gas emission contributions to the observed spectra. To describe the stellar continuum we have used linear combinations of single stellar population (SSP) models based on the MILES library of Sánchez-Blázquez et al. (2006), which will be presented in detail in A. Vazdekis et al. (2006, in preparation). These templates are particularly suited to match the DensePak data, owing to their high spectral resolution $\left(\sigma_{\text {inst }}=42 \mathrm{~km} \mathrm{~s}^{-1}\right)$, and lead to a very good match to the absorption features, such as the prominent line around $6494 \AA$ that is due to calcium and iron. Given the limited number of absorption lines in the $\mathrm{H} \alpha+[\mathrm{N}$ II] region, we have used only a restricted number of SSP templates adopting a Salpeter initial mass function, ages of $t=0.1,0.3,1.0,3.0$, and $10 \mathrm{Gyr}$, and metallicities of $Z=0.5,1.0$, and $1.5 Z_{\odot}$. Such an accurate description of the stellar continuum is crucial to correctly estimate the $\mathrm{H} \alpha$ and [S II] line fluxes toward the center of NGC 7742, where the strength of the gas emission relative to the stellar continuum (and corresponding absorption features) decreases very sharply. Once the emission-line fluxes were measured, the DensePak integrated-flux field was fitted for each of the three pointings taken during the run to derive the position of the galaxy center. The pointings were then shifted to a common central position, adjusted to the same continuum flux level, which varied for each pointing, and then merged on a regular grid matching the sampling of the SAURON data (i.e., $0 " 8 \times 0 " 8$ ). The same process was then used to merge the emission-line fluxes.

\section{RESULTS}

Figure 1 shows the reconstructed images for the distribution of the $\mathrm{H} \alpha$ and [N $\mathrm{II}] \lambda 6583$ emission as measured with DensePak, together with similar maps for the $\mathrm{H} \beta$ and [O III] $\lambda 5007$ fluxes obtained with SAURON. Despite the different designs of these two IFUs and the considerably coarser spatial sampling of DensePak, the overall morphology of the recombination and forbidden lines agrees very well. The rightmost

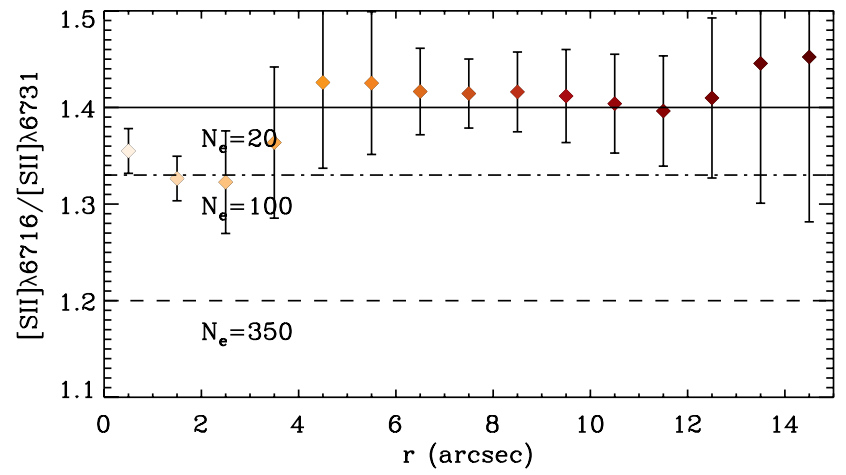

FIG. 2.-Radial variation of the [S II] $\lambda \lambda 6716,6731$ line ratio, which is a tracer of the electron density $N_{e}$. The points show average values of the [S II] line ratio at different radii with error bars indicating the azimuthal variation in this measurement. The horizontal lines indicate the [S II] line ratio corresponding to specific values of $N_{e}$, assuming gas temperature of $T=10^{4} \mathrm{~K}$.

panels of Figure 1 show maps for the $[\mathrm{N}$ II] $\lambda 6583 / \mathrm{H} \alpha$ and [O III] $\lambda 5007 / \mathrm{H} \beta$ line ratios, which will be used to construct the corresponding VO87 diagnostic diagram separating star formation from other sources of ionization.

In the following we constrain the gas density using the ratio of the $[\mathrm{S}$ II] doublet lines $(\S 3.1)$ and present $[\mathrm{N}$ II $] / \mathrm{H} \alpha$ versus $[\mathrm{O}$ III $] / \mathrm{H} \beta$ and $[\mathrm{N}$ II $] /[\mathrm{S}$ II $]$ versus $[\mathrm{O}$ III $] / \mathrm{H} \beta$ diagnostic diagrams to constrain the role of the various ionizing mechanisms in different regions of NGC 7742, as well as the metallicity of the starburst in the circumnuclear regions ( $\$ 3.2)$.

\subsection{Gas Density}

The relative strength of the lines in the [S II] doublet is a strong function of the gas electron density $N_{e}$, only depending weakly on the gas temperature. The observed line ratio can therefore be used to estimate $N_{e}$, although for low and high values of $N_{e}$, the [S II] $\lambda 6716 /[\mathrm{S}$ II] $\lambda 6731$ ratio saturates to values of $\sim 1.4$ and $\sim 0.8$, respectively (Osterbrock 1989). Figure 2 shows, as a function of galactic radius, the ratio of the [S II] lines within the region of NGC 7742 considered for the emission-line diagnostic analysis (see Fig. 1). The [S II] line ratio indicates, on average, $N_{e}=20 \mathrm{~cm}^{-3}$. However, due to the poor sensitivity of this ratio below $N_{e}=100 \mathrm{~cm}^{-3}$, we conclude that the ring is predominantly populated by clouds of very low electronic density, $N_{e}<100 \mathrm{~cm}^{-3}$. Such low values for $N_{e}$ are typical of extragalactic $\mathrm{H}$ II regions but considerably smaller than those observed in the central regions of starburst galaxies, where the typical value is $N_{e} \sim 350 \mathrm{~cm}^{-3}$ within $1 \mathrm{kpc}$ (K01). $N_{e}$ increases toward the center of NGC 7742, although only to $N_{e} \sim 100 \mathrm{~cm}^{-3}$. Following this result, we adopt the lower option of gas densities, $N_{e}=10 \mathrm{~cm}^{-3}$, for the MAPPINGS III photoionization models (K01) that will be used in the next section to further interpret our data.

\subsection{Excitation Mechanisms}

Among the reddening-insensitive diagnostic diagrams introduced by $\mathrm{VO} 87$, the $[\mathrm{O}$ I] $\lambda 6300 / \mathrm{H} \alpha$ versus [O III] $\lambda 5007 / \mathrm{H} \beta$ diagnostic is arguably the best suited to separate OB star excitation from other excitation sources, since the [O I] emission originates in partially ionized regions arising only in the presence of a hard ionizing spectrum. Unfortunately, in NGC 7742 the weak [O I] emission is detected only in the nuclear ring, where the equivalent width of the emission line is the largest. Furthermore, Dopita et al. (2000) showed that the models can- 

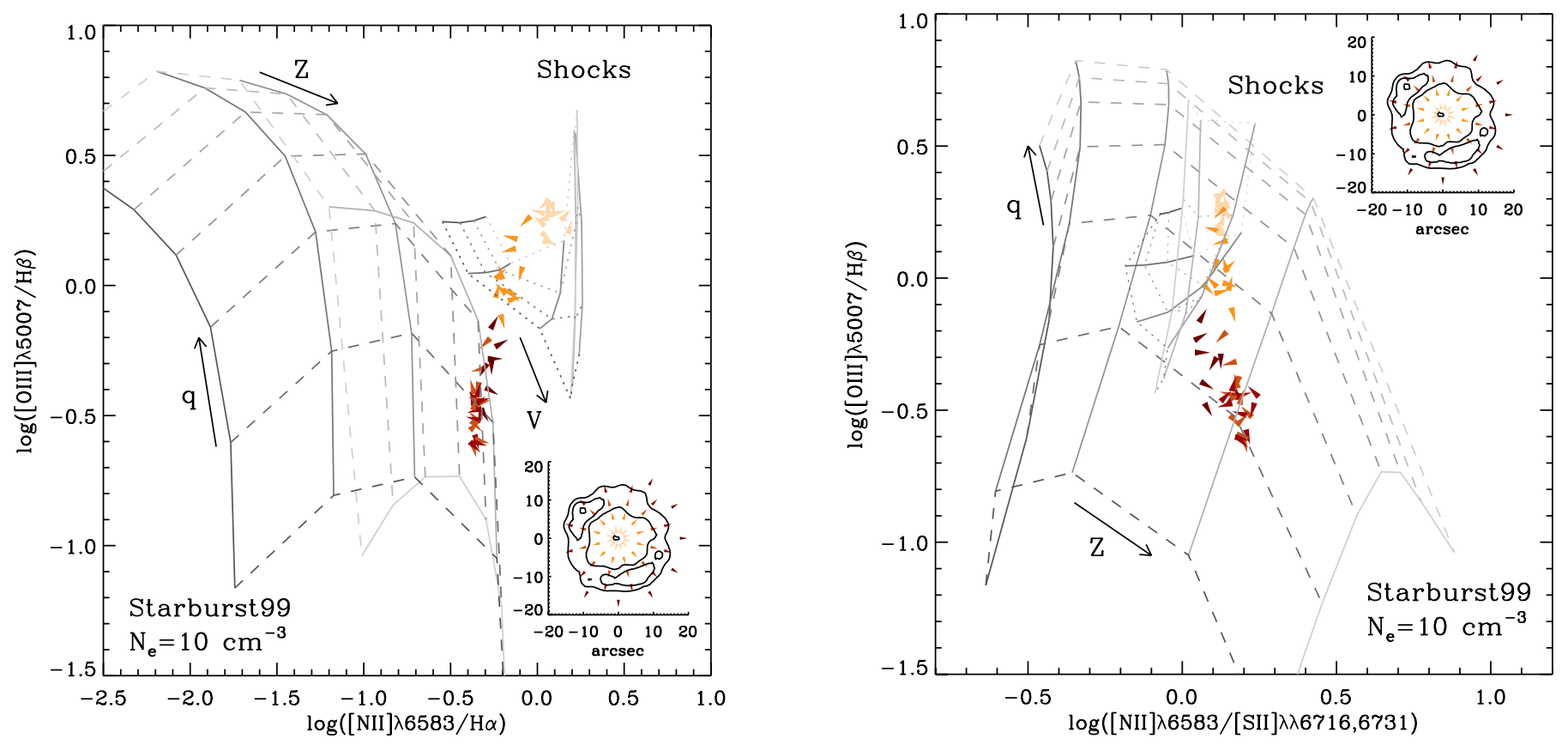

FIG. 3.-Diagnostic diagrams of [O III] $\lambda 5007 / \mathrm{H} \beta$ vs. [N II $] \lambda 6583 / \mathrm{H} \alpha$ (left) and [O III] $\lambda 5007 / \mathrm{H} \beta$ vs. [N II] $]$ 6583/[S II] $\lambda \lambda 6716,6731$ (right). Data from both SAURON and DensePak observations are shown by the filled triangles and were extracted in coronal sections at different distances from the center and at different position angles. In both panels the inset shows the location of these extractions in the galactic frame, with the contours showing the H $\alpha$ circumnuclear ring. The symbols are color-coded from lighter to darker tones for increasing radii and oriented according to the position angle of coronal sections apertures. In each panel the prediction of MAPPINGS III starburst or shock models are shown by the grid of solid and dashed lines or of solid and dotted lines, respectively. For the starburst models, which assume a gas density of $N_{e} \sim 10 \mathrm{~cm}^{-3}$, solid lines of increasingly lighter shades of gray show models of constant metallicity equal to $Z=0.05,0.2,0.4,1.0$, and $2.0 Z_{\odot}$, whereas the dashed lines show models with progressively larger values for the ionization parameter $q=5 \times 10^{6}, 1 \times 10^{7}, 2 \times 10^{7}, 4 \times 10^{7}, 8 \times 10^{7}$, $1.5 \times 10^{8}$, and $3 \times 10^{8} \mathrm{~cm} \mathrm{~s}^{-1}$. Similarly, for the shock models (no precursor $\mathrm{H}$ II region), the gray solid lines show models with increasing shock velocity $V_{s}=$ $150,200,300,500,750$, and $1000 \mathrm{~km} \mathrm{~s}^{-1}$, and the dotted lines models with magnetic parameter $b=0.5,1.0,2.0$, and 4.0.

not distinguish precisely between ionization by hot stars and other sources of ionization using the $[\mathrm{O} \mathrm{I}] / \mathrm{H} \alpha$ and, to some extent, the $[\mathrm{S}$ II] $/ \mathrm{H} \alpha$ diagnostics, as these ratios can be affected by weak shocks from supernovae.

For all these reasons, we thus use the alternative $[\mathrm{N} \mathrm{II}] / \mathrm{H} \alpha$ versus $[\mathrm{O} \mathrm{III}] / \mathrm{H} \beta$ diagnostic diagram, which we show in Figure 3 (left), together with the predictions of MAPPINGS III models for $\mathrm{H}$ II regions in an instantaneous starburst or for clouds excited by shocks. ${ }^{4}$ The starburst models use a spectral energy distribution obtained from Starburst99 (Leitherer et al. $1999)$ and assume a range of metallicity $Z$ for both stars and gas in the starburst and different values of $q$. The shock models assume a range of values for the shock velocity $V_{s}$ and for the magnetic parameter $b$. For a thorough description of these models see Dopita et al. (2000) and Allen et al. (1998).

The $[\mathrm{N} \mathrm{II}] / \mathrm{H} \alpha$ versus $[\mathrm{O} \mathrm{III}] / \mathrm{H} \beta$ diagram (Fig. 3) clearly separates the emission arising in the nuclear ring (red and orange symbols) from emitting regions both outside (brown symbols) and inside the ring (yellow symbols). The line emission from the ring region is consistent with the predictions of the starburst models, whereas the position of the data points measured outside the ring suggests that shocks may play a role in this region, particularly near the inner edge of the ring. As far as the nuclear regions are concerned, however, it is important to keep in mind that models for photoionization by an AGN can predict line ratios very similar to those shown for the shock models (Allen et al. 1998), so that excitation by a central engine cannot be excluded.

Although the $[\mathrm{N}$ II $] / \mathrm{H} \alpha$ versus $[\mathrm{O}$ III $] / \mathrm{H} \beta$ diagnostic diagram

\footnotetext{
${ }^{4}$ Both sets of models were obtained from the MAPPINGS III portal at http://www.ifa.hawaii.edu/ kewley/Mappings.
}

is useful to show the role of different excitation mechanisms in different galactic regions, it cannot generally be used to derive the physical condition of the $\mathrm{H}$ II regions, except in the very low metallicity regime. The models in fact fold around so that any given location in the diagnostic diagram occupied by starburst models with $Z>0.2 Z_{\odot}$ could correspond either to an $\mathrm{H}$ II region of low metallicity and low-ionization parameter or to an $\mathrm{H}$ II region with considerably larger values of $Z$ and $q$. In the combined wavelength range of the DensePak and SAURON observations the best diagnostic to constrain the metallicity of the ring starburst is the $[\mathrm{N}$ II $] /\left[\mathrm{S}_{\mathrm{II}}\right]$ ratio. Rubin et al. (1984) were the first to demonstrate the utility of this line ratio, which serves well to estimate metallicities from slightly subsolar to supersolar values (Kewley \& Dopita 2002). The right panel of Figure 3 shows the location of our data in the $[\mathrm{N}$ II $] /[\mathrm{S}$ II] versus $[\mathrm{O} \mathrm{III}] / \mathrm{H} \beta$ diagnostic diagram, which unequivocally demonstrates that the nuclear ring is predominantly populated by $\mathrm{H}$ II regions with near-solar metallicity. Having learned from the $[\mathrm{N}$ II $] / \mathrm{H} \alpha$ versus $\left[\mathrm{O} \mathrm{III}^{\mathrm{II}}\right] / \mathrm{H} \beta$ diagram that, both inside and outside the ring, sources other than $\mathrm{OB}$ stars may contribute to the gas excitation, we note that the regions outside the ring (brown symbols) seem to approach the shock-model grid from a different direction than the points inside the ring (orange symbols). This suggests that if indeed shocks are contributing to heating and/or ionizing the gas, they may occur under different conditions at the opposite edges of the ring.

\section{DISCUSSION AND CONCLUSIONS}

We have combined two integral-field spectroscopic data sets to study the role of various ionizing mechanisms in powering the gas emission in the central kpc of NGC 7742. Our spatially 
resolved emission-line diagnostic analysis reveals a sharp transition between regions powered by $\mathrm{OB}$ stars in the nuclear ring to LINER-like emission both inside and outside the ring. Comparison with models for shock ionization indicates that shocks can contribute to the gas excitation in these regions, in particular outside the ring. Toward the center photoionization by a central AGN could also be important. The ratio of the [S II] doublet lines indicates low electron density, $N_{e}<100 \mathrm{~cm}^{-3}$, in the ring, where comparison with MAPPINGS III photoionization models shows that the gas and stars have approximately solar metallicity.

NGC 7742 shows a clear signature of past galaxy interaction, namely, the counterrotation of the gas and the stars. Although NGC 7743 accompanies NGC 7742 and is sufficiently massive that it could, in principle, have provided the metal-rich gaseous material observed in the nuclear ring, the large distance between the two galaxies and the morphologically pristine characteristics of both objects rule out a recent interaction. This leads us to consider an alternative scenario, whereby NGC 7742 cannibalized a smaller galaxy, rich in gas of low metallicity. This material was then funneled into the ring where star formation proceeded long enough for the metallicity of the starburst to increase to its present value. Falcón-Barroso et al. (2006) estimated a mass for the ionized gas in NGC 7742 of a few times $10^{6} M_{\odot}$, a lower limit to the total amount of gas in the ring. To reach an oxygen mass fraction of $1 \%$, corresponding to solar metallicity, it would take just tens of Myr to have enough supernovae injecting enriched material in the ring, assuming a star formation rate of a few $M_{\odot} \mathrm{yr}^{-1}$.

We propose that the gas that has fallen into NGC 7742 has reached a rather relaxed, albeit counterrotating, state where massive star formation in the nuclear ring has gradually enriched the metallicity. An analysis of the absorption-line indices in the nuclear ring of NGC 7742 by E. L. Allard et al. (2006, in preparation) supports this by confirming that massive star formation has occurred intermittently there for maybe half a Gyr, in a way very similar to that recently modeled in detail by Allard et al. (2006) for M100. Like the ring in that galaxy, the one in NGC 7742 is thus a stable configuration that can easily enrich its metallicity.

All the observational evidence presented or reviewed here thus strongly suggests that the counterrotating gas came into NGC 7742 through a minor merger event at some time in the history of the galaxy, and that this gas has since reached solar metallicity as a result of the massive star formation in the nuclear ring. Although the counterrotation is unique to the ring host NGC 7742 , a very similar picture of a minor merger at the origin of the nuclear ring in the nonbarred galaxy NGC 278 was proposed on the basis of the disturbed $\mathrm{H}$ I morphology and kinematics in NGC 278 (Knapen et al. 2004).

More constraints on the gas dynamics are needed to construct a detailed chemodynamical model allowing to verify this scenario. Alternatively, deeper spectroscopic data could be used to constrain the metallicity of the gas outside the ring, which according to our model should have a lower metallicity than the nuclear ring material. In addition, deep $\mathrm{H}$ I imaging might reveal the results of the minor merger we postulate for NGC 7742 , possibly in the form of tidal tails or disturbed kinematics. Overall, the techniques applied in this paper can be generalized to other similar systems with composite (AGN+starburst) environments.

We thank Joe Shields, John Barnes, and Uta Fritze for useful discussions. J. H. K. acknowledges support from the Leverhulme Trust in the form of a Leverhulme Research Fellowship. The WHT is operated on the island of La Palma by the Isaac Newton Group in the Spanish Observatorio del Roque de los Muchachos of the Instituto de Astrofísica de Canarias. S. V. is partially supported by NSF grant AST 06-06932.

\section{REFERENCES}

Allard, E. L., Knapen, J. H., Peletier, R. F., \& Sarzi, M. 2006, MNRAS, 371, 1087

Allen, M. G., Dopita, M. A., \& Tsvetanov, Z. I. 1998, ApJ, 493, 571

Bacon, R., et al. 2001, MNRAS, 326, 23

Barden, S. C., \& Wade, R. A. 1988, in ASP Conf. Ser. 3, Fiber Optics in Astronomy, ed. S. C. Barden (San Francisco: ASP), 113

de Zeeuw, P. T., et al. 2002, MNRAS, 329, 513

Dopita, M. A., Kewley, L. J., Heisler, C. A., \& Sutherland, R. S. 2000, ApJ, 542,224

Falcón-Barroso, J., et al. 2006, MNRAS, 369, 529

Fioc, M., \& Rocca-Volmerange, B. 1997, A\&A, 326, 950

Ho, L. C., Filippenko, A. V., \& Sargent, W. L. W. 1997, ApJ, 487, 568

Kewley, L. J., \& Dopita, M. A. 2002, ApJS, 142, 35

Kewley, L. J., Dopita, M. A., Sutherland, R. S., Heisler, C. A., \& Trevena, J. 2001, ApJ, 556, 121 (K01)
Knapen, J. H., Whyte, L. F., de Blok, W. J. G., \& van der Hulst, J. M. 2004, A\&A, 423, 481

Leitherer, C., et al. 1999, ApJS, 123, 3

Mazzuca, L., M, Knapen, J. H., Veilleux, S., \& Regan, M. W. 2006, ApJ, submitted

Osterbrock, D. E. 1989, Astrophysic of Gaseous Nebulae and Active Galactic Nuclei (Mill Valley: University Science Books)

Pogge, R. W., \& Eskridge, P. B. 1993, AJ, 106, 1405

Rubin, V. C., Ford, W. K., \& Whitmore, B. C. 1984, ApJ, 281, L21

Sánchez-Blázquez, P., et al. 2006, MNRAS, in press (astro-ph/0607009)

Sarzi, M., et al. 2006, MNRAS, 366, 1151

Sil'chenko, O. K., \& Moiseev, A. V. 2006, AJ, 131, 1336

Veilleux, S., \& Osterbrock, D. E. 1987, ApJS, 63, 295 (VO87) 